\title{
Mesenchymal stem cells in the reconstruction surgery of the supraspinatus muscle lesions
}

\begin{abstract}
Background: The objective of the study was to demonstrate the effect of concentrated bone marrow stem cells (MSCs) to the healing of sutured tendon of the supraspinatus muscle in comparison to the same procedure performed without MSCs. We have postulated the hypothesis that MSCs enhance the healing of the sutured supraspinatus tendon to its humeral footprint.
\end{abstract}

Methods and findings: None of the patients had to be excluded from our prospective randomized study. 50 patients met the indication criteria for isolated supraspinatus surgery. 25 patients (Group I) received MSCs into the supraspinatus footprint. 25 patients (Group II) was treated without the use of MSCs. Patients were examined pre-operatively, 6, 12 and 24 months after the surgery and assessment included physical examination, visual analogue scale (VAS), ASES (American Shoulder and Elbow Surgeons Standardized Shoulder Assessment Form) and Constant score. Patients underwent MRI 2 years postoperatively. Findings were compared with the preoperative results.

We have found no statistically significant differences between both groups pre-operatively. After a 24-month follow-up period there were significant differences observed in all clinical outcomes. When compared results between both groups two years postoperatively, $p$-value $(p=0.05)$ for the VAS score was 0.0176 and for the Constant score 0.0355 . The only statistically non-significant was $p$-value for the ASES score comparing results from both groups at two years follow-up ( $p=0.085)$. The MRI findings at 2 years showed fully healed tissue of the rotator cuff tendon in 17 patients in the Group I, but only in 9 patients in the Group II.

Conclusions: We have accepted the hypothesis that MSCs enhance the healing of the sutured supraspinatus tendon to its humeral footprint.

Keywords: Bone marrow stem cells $\boldsymbol{~}$ rotator cuff lesion

Submitted: 04 August 2017; Accepted: 28 August 2017; Published online: 02 September 2017 most common diseases of the shoulder joint and often results in clinically significant pain and dysfunction $[1,2]$. Successful treatment of rotator cuff tears is often complicated by a reduced tendency for healing, even if the proper surgical treatment is used [3]. Rotator cuff tendon is a tissue known to have a poor vascularisation and a relatively low tendency to heal when torn. The poor vascularisation causes insufficient migration of cells involved in the healing process of tendon rupture
Petr Šmíd ${ }^{1,2}$, Martin Komzák*, Radek Hart ${ }^{1}$, Libor Paša ${ }^{2}$

'Department of Orthopaedic and Traumatologic Surgery Hospital Znojmo MU Dr. Jana Jánského 11

Znojmo, 669 02, Czech Republic ${ }^{2}$ Department of Traumatologic Surgery University of Masaryk at Brno, Faculty of Medicine Ponávka 6 Brno, 602 00, Czech Republic *Author for correspondence: m.komzak@seznam.cz martin.komzak@nemzn.cz

\section{Introduction}

Damage of the rotator cuff is one of the 
[4]. Histological studies show, that the tendons of the rotator cuff do not heal by the regeneration of the original tendon (fibrocartilage insertion), but by the formation of scar tissue with a high content of collagen III $[5,6]$.

Treatment of ruptured rotator cuff tendons is not always successful. The use of anchors has facilitated tendon fixation to the bone [7], non-absorbable sutures significantly increased resistance of this suture [8]. Nevertheless, none of these advances has brought significant improvement to the quality of healing and postoperative results, especially in patients with large rotator cuff injuries $[9,10]$. In large lesions there are often unsatisfactory long term results and many studies show a high percentage of re-ruptures (11-94\%) [11-13]. High rates of the tendon injury and re-rupture accelerate investigations of methods modulating tendons healing. The use of tissue-derived stem cells grows in popularity because of their relatively easy acquisition, application and their potential multipotency, enabling them to differentiate and participate in the healing of various musculoskeletal tissues, including tendons [14,15]. Mesenchymal stem cells (MSCs) are undifferentiated cells that have the ability to divide (proliferate) and transform (differentiate) to another cell type. Because of this potential these cells can accelerate the healing process at the tendon- bone interface [16]. In adults, these multipotent cells originate from various mesenchymal tissues such as bone marrow, perivascular tissues, blood, tendon, muscle and adipose tissue [17].

The aim of our study was to accept or refuse the hypothesis that the MSCs of an adult can improve the healing process at the bone / tendon interface of the rotator cuff.

\section{Methods}

The prospective randomized study was carried out at orthopaedic and traumatology, haematology and radiology departments between 2012 and 2014. The study included 50 patients, who were divided into two groups, each of 25 patients. Group of patients I, who received the concentrate of MSCs into the tendonbone contact point, consisted of 7 women and 18 men (mean age 51, 2 years) and Group II (without the use of MSCs during the surgery) consisted of 15 women and 10 men (mean age 50, 5 years). Division of patients into groups was performed using Random Number Generator Software 7.0.

In order for the patient to be admitted to the study, had to meet the following conditions: male or female aged 18-60 years, supraspinatus tendon lesion greater than $50 \%$ of its width (no previous trauma to the examined shoulder joint), confirmation of the tendon tear by the clinical examination and preoperative MRI arthrography, signed informed consent, the internal state permitting the surgery. Exclusion criteria were: previous surgery of the shoulder joint, arthropathy of the shoulder joint grade 3-5 according to Hamada classification [18], fatty degeneration of the cuff muscle (Goutallier 3 and 4) [19], atrophy of the supraspinatus muscle according to the Thomazeau classification grade II and III [20] (grade II patients according to the Thomazeau classification had to be eliminated, because the occupation ratio of the supraspinatus fossa by the supraspinatus muscle 0,60-0,40 indicates the degree of atrophy increasing the risk of tendon rerupture), retraction of the supraspinatus muscle tendon Patte III [21], pregnancy, active infection at the surgical site or elsewhere in the body, osteomyelitis, sepsis, history of diabetes, steroids use, chemotherapy, use of NSAIDs (NSAIDs are supposed to reduce tissue healing properties) [22-24], radiation, hepatitis, HIV, hemato/oncological diseases, alcohol abuse, drug abuse, cigarettes, medical condition that affects the growth of bone or connective tissue (osteomalacia or other metabolic bone disease), vascular insufficiency, operated extremity muscle atrophy, neuromuscular disease. All of radiographs/MRI/CT results were evaluated by an experienced radiologist specialized in musculoskeletal pathologies that were blinded to the both groups of patients.

Prior to entering the study, patients were informed by the consent produced by the Ethical Committee of local hospital and were randomly assigned to one of the treatment methods with use of the computer software by the method $1: 1$.

Before the surgery itself, patients had to complete three questionnaires, with VAS score, ASES score (American shoulder and elbow surgeons standardized shoulder assessment form) [25] and the Constant score [26]. Further clinical control with completing these questionnaires was 6 months after surgery, 1 year after surgical treatment and 2 years postoperatively. Two years after the surgery MRI examination of the shoulder joint was performed. With VAS score patients subjectively rated the pain level at 6 grade scale from 0-5 where 0 represented no pain and 5 excruciating pain. ASES questionnaire consists of two parts. The first of them is a questionnaire filled in by the patient. This includes a VAS for pain and instability and assessment of capability to perform activities of daily living (ADL). The second part that was completed by a doctor is 
represented by the objective evaluation of ROM (range of motion), specific physical characteristics, stability and strength. Final score for the shoulder was then derived from the visual analogue scale for pain $(50 \%)$ and the general score for ADL (50\%). Constant score represents another system for evaluating the condition of the shoulder joint, subjectively from the patient's perspective; the second part is an objective assessment of range of motion and muscle strength performed by the physician. All of the outpatient clinical evaluations during the study were performed by the orthopaedic surgeon blinded to the both groups of patients.

The actual therapy was as follows: open surgery was preceded by a standard diagnostic arthroscopy, which was performed from the posterior portal for optics and anterior portal for arthroscopic instruments. The rotator cuff tendons were evaluated from both the intra-articular and sub acromial side. To be able to evaluate cuff tendon tear arthroscopically (Figure 1), we used a special arthroscopic hook calibrated in 1 $\mathrm{mm}$. To evaluate the rupture shape, the classification of Ellman - Gartsman [27] was used; where type 1 is a rupture of the crescent-shape, type 2 inverted L, type $3 \mathrm{~L}$-shape, type 4 trapezoidal shapes, and the type 5 - a massive rupture and tendon retraction. Surgical treatment of injured tendons of the rotator cuff was performed in the beach chair position of the patient, by the deltoid muscle split approach (Figure 2). After careful evaluation of the size and location of the tendon

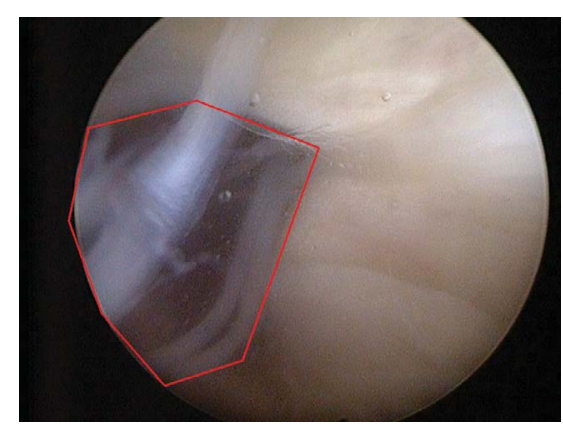

Figure 1: Rotator cuff lesion diagnostics

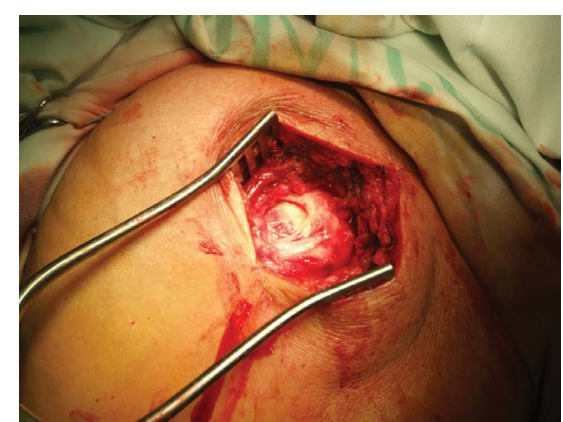

Figure 2: Deltoid-split approach to the shoulder joint tear the aspiration of $10 \mathrm{ml}$ of bone marrow blood from the humeral head into a $20 \mathrm{~mL}$ syringe prefilled with $0.5 \mathrm{ml}$ of heparin with the Yamshidi needle was done (Figure 3). A maximum of 3 millilitres of marrow blood was aspirated from each bone puncture. Centrifugation of the aspirate was done in $10 \mathrm{ml}$ test-tube Monovette filled with citrate (Figure 4). We chose a centrifuge regime of gradient 15 minute centrifugation at $200 \mathrm{~g}$ RCF (relative centrifugal force). After the centrifugation the tube content was divided into three main parts. The most superficial layer consists of the plateletrich plasma (PRP), the middle layer of the buffy coat with the dominant presence of leukocytes, and the lowest layer is formed by sedimented erythrocytes. A collection of the buffy coat was performed by using of sterile laboratory pipette (Figure 5). MSCs represented 0.01 to $0.02 \%$ of all nuclear elements of the buffy coat that is about $10 \times 10^{6} / 1$. The MSCs concentrate

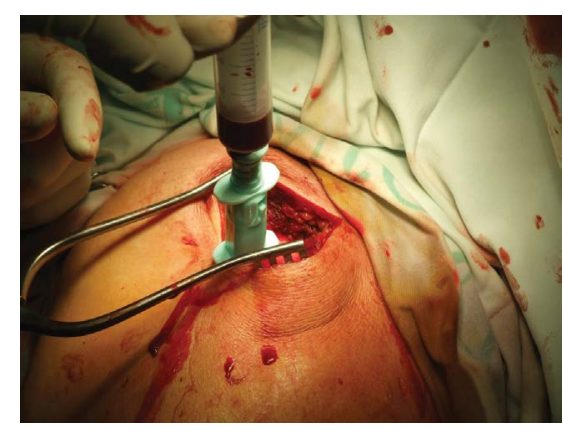

Figure 3: Medulary blood aspiration

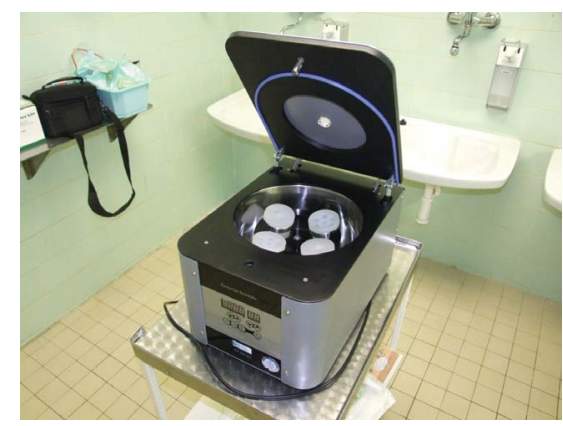

Figure 4: Centurion Scientific C2 centrifuge

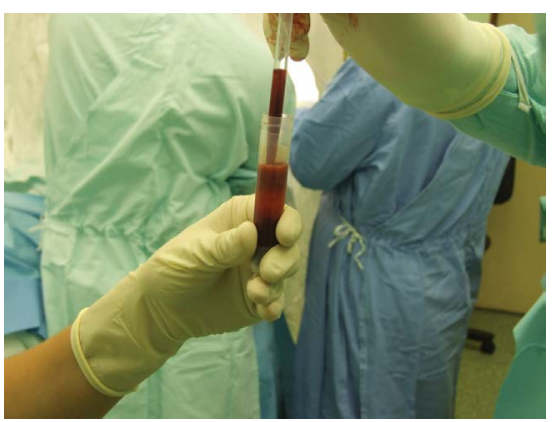

Figure 5: Buffy coat acquisition 
(similar to synovial fluid in consistency and colour) was impregnated into the collagen patch Hypro-Sorb F (Otrokovice, Czech Republic) of dimensions $1 \times 2$ $\mathrm{cm}$ (Figure 6). The collagen patch as a carrier of the MSCs concentrate was used to ensure its retention in desired place. This material was then inserted between the tendon and bone, into the place of re-fixing of the rotator cuff tendons (footprint) before tightening the threads of the anchors (Figure 7). Anchoring the tendon to the bone was made by a double row method with use of absorbable fixation material Bio Composite Corkscrew (Arthrex, Munich, Germany) (Figure 8). In all cases treated by us the tendon was fixed in place of its original insertion without any tension and without the need of abduction of the arm above 60 degrees. All patients underwent Neer acromioplasty to gain more space subacromially. This procedure was followed

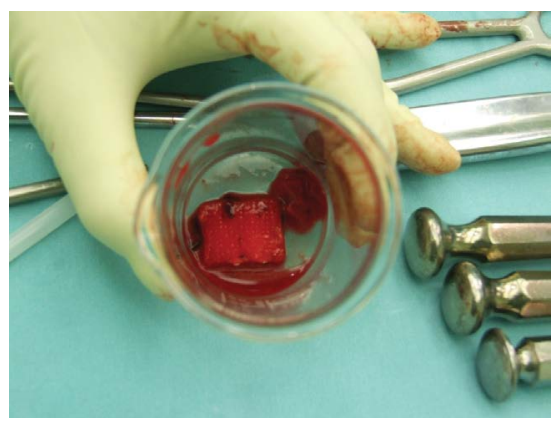

Figure 6: Collagen patch with MSCs

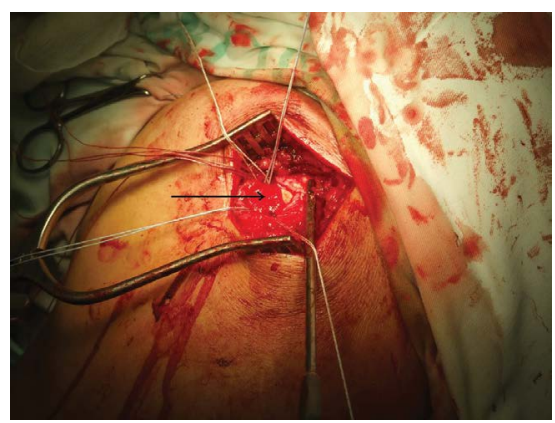

Figure 7: Arrow indicating collagen patch impregnated with MSCs in the supraspinatus footprint

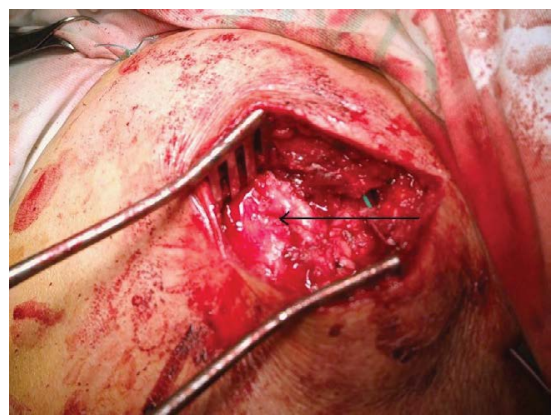

Figure 8: Arrow indicating repaired rotator cuff defect

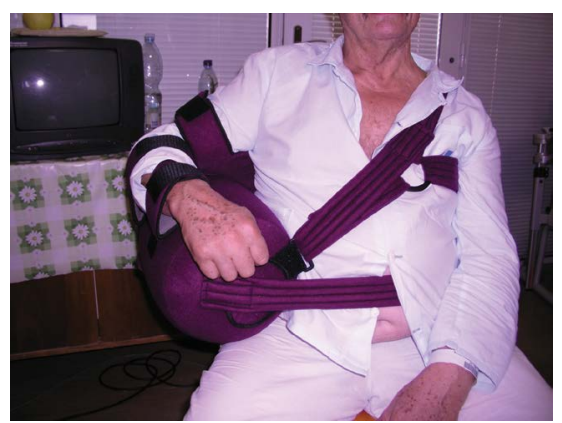

Figure 9: Abduction orthosis applied

by the wound closure in all anatomical layers, sterile dressing of the wound and by the abduction orthosis application (Figure 9). All patients were operated by the same orthopaedic surgeon (lead author of the article) with the same surgical technique. Open surgery instead of arthroscopy was chosen because of the safer fixation of the MSCs carrier under the re-inserted tendon and to reduce the risk of washout of the MSCs concentrate from the carrier during the arthroscopy.

Patients from the Group II were treated by the identical surgical method as in Group I, except the aspiration of bone marrow from the humeral head and subsequent application of the carrier saturated with MSCs into the tendon/bone contact.

The abduction brace was worn for 4 weeks. Passive motion above the shoulder level under the guidance of a physiotherapist was initiated the first day postoperatively. After removing the fixation, an active part of rehabilitation began. That is full range of motion done actively 6 weeks after surgery. Full load of the operated limb was allowed 12 weeks after the surgery.

Outpatient follow-up (performed by orthopaedic surgeon blinded to both groups of patients) took place according to the schedule mentioned above. To check the integrity of the tendon at the point of insertion into the greater tuberosity of the humerus, MRI of the shoulder joint was performed 2 years postoperatively. Both, preoperative and postoperative MRI examinations were performed with a $1.5 \mathrm{~T}$ highfield scanner, in sagittal, coronal, and axial planes, in T1- and T2-weighted fat-saturated sequences (MRI arthrography using gadollinium as a contrast medium). All of MRI results were evaluated by an experienced radiologist specialized in musculoskeletal pathologies. He evaluated tendon integrity, presence and localisation of complete retear, tendon and muscle borders, and presence of abnormal artifacts.

The primary hypothesis of this study was that the use of mesenchymal stem cells in the supraspinatus tendon 
reconstruction surgery will have a positive effect on both subjective and objective postoperative evaluation, as well as to reduce the number of tendon re-ruptures compared to the patient's population in which mesenchymal stem cells were not used. All data were statistically analyzed with STATISTICA 9.0 program. To compare the results of the of the VAS score, the Constant score, and the ASES score preoperatively and one year after the surgery Student's t- test was used, to compare the incidence of re-ruptures between both groups of patients 2 years postoperatively Chi-square test was used. The significance level was determined at $\mathrm{p}<0.05$. Patients were randomly assigned to one of the treatment possibilities with use of the Random Number Generator Software 7.0 by the method 1:1

\section{Results}

Incidence of the supraspinatus tendon tear shape in both groups of patients according to Ellman Gartsman classification is shown in Table 1. Results of the perioperative rupture width measurement are summarized in Table 2. Preoperative clinical scores evaluation results for both groups and same measurements from 24-month follow-up are summarized in Table 3. There were no statistically significant differences between both groups pre- operatively. According to the Student's t- test $(\mathrm{p}=0.05)$ comparing the results of the VAS score, the Constant score, and the ASES score

Table 1: Incidence of the tendon rupture shape according to Ellman- Gartsman classification

\begin{tabular}{|lccccc|} 
& Type 1 & Type 2 & Type 3 & Type 1 & Type 1 \\
\hline Group I & 18 & 2 & 3 & 2 & 0 \\
\hline Group II & 17 & 3 & 3 & 2 & 0 \\
\hline
\end{tabular}

\begin{tabular}{|ccccc|}
\hline \multicolumn{5}{c|}{ Table 2: Tendon rupture width } \\
\hline & $\begin{array}{c}\text { Mean } \\
(\mathbf{~ m m})\end{array}$ & $\begin{array}{c}\text { Range } \\
(\mathbf{m m})\end{array}$ & $\begin{array}{c}\text { Median } \\
(\mathbf{m m})\end{array}$ & $\begin{array}{c}\text { Standard } \\
\text { deviation }\end{array}$ \\
\hline Group I & 12,33 & $6-16$ & 12 & 2,98 \\
\hline Group II & 13,11 & $8-16$ & 13 & 2,38 \\
\hline
\end{tabular}

between both groups two years postoperatively, p-value for the VAS score was 0.0176 , for the Constant score 0.0355 and for the ASES score 0.0846. MRI analysis after a 24-months follow-up period demonstrated re-ruptures in rotator cuff tendon attachment in 16 patients (64\%) in the Group II, compared to that only in 8 patients (32\%) in the Group I ( $\mathrm{p}$-value=0.02354). All of the reruptures have been localized at the point of reinsertion of rotator cuff tendons to the greater tuberosity (at the footprint). Only in $25.0 \%$ of patients from Group I and in $37.5 \%$ of patients from Group II with tendon re-rupture there was a positive correlation between subjective and objective postoperative findings and occurrence of the tendon re-rupture.

\section{Discussion}

One of the most important findings of our work is that the use of mononuclear autologous MSCs during the rotator cuff surgery is a safe procedure that provides better functional outcomes than the standard suture methods. We have accepted the hypothesis that MSCs enhance the healing of the sutured supraspinatus tendon to its humeral footprint. Results of the p-value for the VAS score and for the Constant score were statistically significant $(\mathrm{p}<0.05)$. The only statistically non-significant was $p$-value for the ASES score comparing results from both groups at two years follow-up $(\mathrm{p}=0.085)$. However, the outcome of the ASES score is determined by the subjective evaluation of the patient's pain (maximum 50 points) and partly subjective assessment of activities of daily living (max 50 points). The ASES score value does not count with the objective active range of motion evaluated by the physician as for example the Constant score does. The well-known truth is that clinical results (for example ASES score) do not correlate with the real re-rupture rate. In our study the average ASES score in 2 year follow-up for the Group I was 87.6 points (that is $87.6 \%$ from maximum of 100 points), for the Group II it was 77.6 points $(77.6 \%)$. On the other hand, we found $68 \%$ well healed rotator cuff tendons in the Group

\begin{tabular}{|c|c|c|c|c|c|c|c|c|c|c|c|c|}
\hline & \multirow[b]{2}{*}{ Mean } & \multicolumn{3}{|c|}{ VAS } & \multicolumn{4}{|c|}{ ASES } & \multicolumn{4}{|c|}{ Constant } \\
\hline & & Range & Median & $\begin{array}{l}\text { Stand. } \\
\text { deviation }\end{array}$ & Mean & Range & Median & $\begin{array}{c}\text { Stand. } \\
\text { deviation }\end{array}$ & Mean & Range & Median & $\begin{array}{c}\text { Stand. } \\
\text { deviation }\end{array}$ \\
\hline Group I preop. & 3,04 & $1-4$ & 3,0 & 0,77 & 45,04 & $22-75$ & 38,0 & 19,63 & 43,04 & $21-68$ & 52,0 & 17,15 \\
\hline $\begin{array}{l}\text { Group I } \\
\text { postop. }\end{array}$ & 0,60 & $0-3$ & 0,0 & 0,90 & 87,60 & $30-100$ & 97,0 & 19,73 & 78,28 & $37-95$ & 85,0 & 15,41 \\
\hline$P$ value & \multicolumn{4}{|c|}{$2,77 \times 10^{-10}$} & \multicolumn{4}{|c|}{$6,48 \times 10^{-8}$} & \multicolumn{4}{|c|}{$2,25 \times 10^{-7}$} \\
\hline Group II preop. & 3,08 & $1-5$ & 3,0 & 0,80 & 48,12 & $17-77$ & 50,0 & 19,08 & 45,12 & $22-72$ & 43,0 & 16,47 \\
\hline $\begin{array}{l}\text { Group } \\
\text { Ilpostop. }\end{array}$ & 1,28 & $0-3$ & 1,0 & 1,25 & 77,60 & $27-100$ & 85,0 & 24,33 & 67,96 & $21-86$ & 78,0 & 18,96 \\
\hline P value & \multicolumn{4}{|c|}{$1,70 \times 10^{-6}$} & \multicolumn{4}{|c|}{0,000157} & \multicolumn{4}{|c|}{0,000101} \\
\hline
\end{tabular}


I, but only $36 \%$ of the rotator cuff tendons without rerupture in the Group II. The difference of results when evaluated clinically and when evaluated according to the real re-rupture rate is significant.

One promising area of research in cellular therapy of last two decades is the possibility to use MSCs in the treatment of tendon injuries. The great advantage of using MSCs is their ability of multipotent differentiation, availability, relatively simple material handling, and their immunological properties [28,29]. Use of autologous MSCs for the treatment of tendons, muscles, and cartilage is currently the subject of numerous research studies in experimental animal models with not entirely consistent results [30,31]. Well known method is the use of MSCs in tendinopathy of racehorses, particularly in the treatment of partial tendon injury where the therapy with MSCs have a significant impact on the ability of animals to return to the racing [32]. Schnabel et al. [33] presented an in vivo study at the horses, in which the partial rupture of musculus flexor digitorum superficialis tendon was formed, and into which the concentrate of MSCs $10 \times 10(6)$ was applied. Ultrasound control was performed at 0, 2, 4, 6 and 8 weeks after the application. In the eighth week, horses were sacrificed and treated tendons were tested mechanically, biochemically and histologically. There was an increased histological quality of the tendons treated with MSCs compared with the control group without treatment by MSCs. Kida et al. [34] have demonstrated in their study on a rat model, that MSCs have ability to migrate from the bone marrow of the greater humeral tuberosity, when predrilled, into the tendon and potentiate healing on the tendon/bone interface.

To this date there are only few human clinical studies using MSCs from bone marrow. Level IV study presented by Ellera-Gomes et al. [9] evaluated the clinical use of non-cultured mononuclear cells that have been after the closure of the lesion injected between the tendon and bone in the "footprint". Mononuclear cells have been in this study collected before the surgery from the iliac crest, processed by Ficoll-Hypaque density gradient, and then resuspended in saline solution enriched with $10 \%$ autologous serum. In this study 14 patients showed improvement in clinical scores and good repair integrity observed on MRI on average 12 months after the rotator cuff tear surgery. In contrary to our work authors of this study haven't compared their results with the control group.

Clinical study presented by Hernigou et al. in 2014 compared the treatment of the rotator cuff muscles tendon ruptures by single-row method using MSCs and without the use of MSCs. During the ten years follow up, authors have demonstrated, that the use of MSCs has a significant effect on speeding up the healing of rotator cuff tendons tears compared to the control group, as well as having a significant impact on reducing the number of re-ruptures of tendons in patients, where the method of MSCs was used [35].

One of the last studies available dealing with the use of human MSCs was presented by Park et al. [36]. In this study, authors investigated the therapeutic effects of ultrasound-guided human umbilical cord blood derived injection to regenerate a full-thickness subscapularis tendon tear in a rabbit model by analyzing morphology and histology of the injected tendon and motion analysis of the rabbit's activity. At 4 weeks after the MSC injection, 7 of the 10 full-thickness subscapularis tendon tears were only partial-thickness tears, and 3 remained full-thickness tendon tears. The tendon tear size and walking capacity at 4 weeks after the MSC injection were significantly improved compared with the same parameters immediately after tendon tear. Histology revealed that MSCs induced regeneration of the rotator cuff tendon tear and that the regenerated tissue was predominantly composed of type I collagens.

During the evaluation of the strength, biomechanical properties and histological image of healed tendon was also considered the usage of various cell carriers, such as fibrin carriers and various collagen nets to ensure the maintenance of cell prepares at the lesion site. Most studies evaluate cellular contribution to healing of tendon as significant. In terms of technology application, amount and concentration of the MSCs is still no clear consensus [37-40].

The results of our study show that the use of the MSCs concentrate in patients undergoing the surgery for the rotator cuff tendon rupture gives promising results compared with patients who underwent identical surgery, but without the use of the MSCs concentrate. Our results also indicate that this procedure is safe enough to be used in clinical praxis and therefore these results reinforce the importance of further research to better investigate the use of this biological approach to enhance tissue quality in affected rotator cuff tendons. We have confirmed our hypothesis, that the MSCs have a positive effect on the subjective and objective post-operative status and on the healing ability of the tendons at the point of its insertion to the bone.

\section{Acknowledgements}

Not applicable 


\section{Competing interests}

The authors declare that they have no competing interests.

\section{Funding}

No funding had role in the design of the study.

\section{Executive summary}

Background: The objective of the study was to demonstrate the effect of concentrated bone marrow stem cells (MSCs) to the healing of sutured tendon of the supraspinatus muscle in comparison to the same procedure performed without MSCs. We have postulated the hypothesis that MSCs enhance the healing of the sutured supraspinatus tendon to its humeral footprint.

Methods and findings: None of the patients had to be excluded from our prospective randomized study. 50 patients met the indication criteria for isolated supraspinatus surgery. 25 patients (Group I) received MSCs into the supraspinatus footprint. 25 patients (Group II) was treated without the use of MSCs. Patients were examined pre-operatively, 6, 12 and 24 months after the surgery and assessment included physical examination, visual analogue scale (VAS), ASES (American Shoulder and Elbow Surgeons Standardized Shoulder Assessment Form) and Constant score. Patients underwent MRI 2 years postoperatively. Findings were compared with the preoperative results.

We have found no statistically significant differences between both groups pre-operatively. After a 24 month follow-up period there were significant differences observed in all clinical outcomes. When compared results between both groups two years postoperatively, $p$-value $(p=0.05)$ for the VAS score was 0.0176 and for the Constant score 0.0355 . The only statistically nonsignificant was $p$-value for the ASES score comparing results from both groups at two years follow-up ( $p=0.085)$. The MRI findings at 2 years showed fully healed tissue of the rotator cuff tendon in 17 patients in the Group I, but only in 9 patients in the Group II.

Conclusions: We have accepted the hypothesis that MSCs enhance the healing of the sutured supraspinatus tendon to its humeral footprint.

\section{References}

1. Hawkins RJ, Kennedy JC. Impingement syndrome in athletes. Am J Sports Med 8: 151-158 (1980).

2. Šmíd P, Hart R, Puskeiler M. Tangent sign - a reliable predictor of risk for tendon re-rupture in rotator cuff repair. Acta Chir orthop Traum Cech 81: 227-232 (2014).

3. Galatz LM, Ball CM, Teefey SA, Middleton WD, Yamaguchi K. Rotator cuff tears: why do we repair them? The outcome and repair integrity of completely arthroscopically repaired large and massive rotator cuff tears. J Bone Joint Surg Am 86: 219-224 (2004).

4. Benjamin M, Ralphs JR. Tendons and ligaments - an overview. Histol Histopathol 12: 1135-1144 (1997).

5. Galatz LM, Sandell LJ, Rothermich SY, Das R, Mastny A, Havlioglu $\mathrm{N}$, et al. Characteristics of the rat supraspinatus tendon during tendon-to-bone healing after acute injury. $J$ Orthop Res 24: 541-550 (2006).

6. Rodeo SA, Arnoczky SR, Torzilli PA, Hidaka C, Warren RE. Tendon-healing in a bone tunnel. A biomechanical and histological study in the dog. J Bone Jt Surg 75: 1795-1803 (1993).

7. Goble EM, Somers WK, Clark R, Olsen RE. The development of suture anchors for use in soft tissue fixation to bone. $A m J$ Sports Med 22: 236-239 (1994).

8. Barber FA, Coons DA, Ruiz-Suarez M. Cyclic load testing of biodegradable suture anchors containing 2 high-strength sutures. Arthrosc 23: 355-360 (2007).

9. Ellera Gomes JL, Da Silva RC, Silla LM, Abreu MR, Pellanda R. Conventional rotator cuff repair complemented by the aid of mononuclear autologous stem cells. Knee Surg Sports Traumatol Arthrosc 20: 373-377 (2012).
10. Jo CH, Shin JS. Cross-sectional area of the supraspinatus muscle after rotator cuff repair: an anatomic measure of outcome. J Bone Joint Surg Am 95: 1785-1791 (2013).

11. Oh JH, Kim SH, Ji HM, Jo KH, Bin SW, Gong HS. Prognostic factors affecting anatomic outcome of rotator cuff repair and correlation with functional outcome. Arthrosc 25: 30-39 (2009).

12. Mihata T, Watanabe C, Fukunishi K, Ohue M, Tsujimura T, Fujiwara K, et al. Functional and structural outcomes of singlerow versus double-row versus combined double-row and suturebridge repair for rotator cuff tears. A. J Sports Med 39: 20912098 (2011).

13. Carbonel I, Martínez AA, Aldea E, Ripalda J, Herrera A. Outcome and structural integrity of rotator cuff after arthroscopic treatment of large and massive tears with double row technique: a 2-year followup. Adv Orthop 914148: (2013).

14. Chong AK, Ang AD, Goh JC, Hui JH, Lim AY, Lee EH, et $a l$. Bone marrow-derived mesenchymal stem cells influence early tendon-healing in a rabbit achilles tendon model. J Bone Join. Surg Am 89: 74-81 (2007).

15. Chong AK, Chang J, Go JC. Mesenchymal stem cells and tendon healing. Front Biosci (Landmark Ed) 14: 45984605 (2009).

16. Gulotta LV, Kovacevic D, Packer JD, Deng XH, Rodeo SA. Bone marrow-derived mesenchymal stem cells transduced with scleraxis improve rotator cuff healing in a rat model. Am J Sports Med 39: 1282-1289 (2009).

17. Caplan AI. Adult mesenchymal stem cells for tissue engineering versus regenerative medicine. J Cell Physiol 213: 341-347 (2007). 
18. Hamada K, Fukuda H, Mikasa M, Kobayashi Y. Roentgenographic findings in massive rotator cuff tears. A longterm observation. Clin Orthop Relat Res 254: $92-96$ (1990).

19. Goutallier D, Postel J, Bernageau J, Lavau L, Voisin M. Fatty muscle degeneration in cuff ruptures. Pre-and postoperative evaluation by CT scan. Clin Orthop Relat Res 304: 78-83 (1994).

20. Thomazeau H, Boukobza E, Morcet N, Chaperon J, Langlais F. Prediction of rotator cuff repair results by magnetic resonance imaging. Clin Orthop Relat Res 344: 275-283 (1997).

21. Patte D. Classification of rotator cuff lesions. Clin Orthop Relat Res 254: 81-86 (1990).

22. Riley G. Tedinopathy-from basic science to treatment. Nat Clin Pract Rheumatol 4: 82-89 (2008).

23. Elder CL, Dahners LE, Weinhold PS. A cyclooxygenase-2 inhibitor impairs ligament healing in the rat. Am J Sports Med 29: 801-805 (2001)

24. Ferry ST, Dahners LE, Afshari HM, Weinhold PS. The effects of common anti-inflammatory drugs on the healing rat patellar tendon. Am J Sports Med 35: 1326-1333 (2007).

25. Richards RR, An KN, Bigliani LU, Friedman RJ, Gartsman GM, Gristina AG, Iannotti JP, Mow VC, Sidles JA, Zuckerman JD. A standardized method for the assessment of shoulder function. J Shoulder Elbow Surg 3: 347-352 (1994).

26. Constant CR, Murley AHG. A clinical method of functional assessment of the shoulder. Clin Orth Rel Res 214: 160-164 (1987).

27. Ellman H. Arthroscopic shoulder surgery and related disorders. Philadelphia: Lea and Febiger 98-119 (1993).

28. Zheng YH, Xiong W, Su K, Kuang SJ, Zhang ZG. Multilineage differentiation of human bone marrow mesenchymal stem cells in vitro and in vivo. Exp Ther Med 5: 1576-1580 (2013).

29. Pittenger MF, Mackay AM, Beck SC, et al. Multilineage potential of adult human mesenchymal stem cells. Sci 284: 143-147 (1999).

30. Urdzikova L, Sedlacek R, Suchy T, Ameroni T, Ruzicka J, Lesný $\mathrm{P}$, et al. Human multipotent mesenchymal stem cells improve healing after collagenase tendon injury in the rat. Biomed Eng 13: 42 (2014)
31. Yokoya S, Mochizuki Y, Natsu K, Omae H, Nagata Y, Ochi M. Rotator cuff regeneration using a bioabsorbable material with bone marrow-derived mesenchymal stem cells in a rabbit model. Am J Sports Med 40: 1259-1268 (2012).

32. Pacini S, Spinabella S, Trombi L, Fazzi R, Galimberti S, Dini F, et al. Suspension of Bone Marrow-Derived Undifferentiated Mesenchymal Stromal Cells for Repair of Superficial Digital Flexor Tendon in Race Horses. Tissue Eng 13: 2949-2955 (2007).

33. Schnabel LV, Lynch ME, van der Meulen MC, Yeager AE, Kornatowski MA, Nixon AJ. Mesenchymal stem cells and insulin-like growth factor-I gene-enhanced mesenchymal stem cells improve structural aspects of healing in equine flexor digitorum superficialis tendons. J Orthop Res 27: 1392-1398 (2009).

34. Kida Y, Morihara T, Matsuda K, Kajikawa Y, Tachiiri H, et al. Bone marrow-derived cells from the footprint infiltrate into the repaired rotator cuff. J Shoulder Elbow Surg 22: 197-205 (2013).

35. Hernigou P, Flouzat Lachaniette $\mathrm{CH}$, Delambre J, Zilber $S$, Duffiet $\mathrm{P}$, et al. Biologic augmentation of rotator cuff repair with mesenchymal stem cells during arthroscopy improves healingand prevents further tears: a case-controlled study. Int Orthop 38: 1811-1818 (2014).

36. Park GY, Kwon DR, Lee SC. Regeneration of fullthickness rotator cuff tendon tear after ultrasound-guided injection with umbilical cord blood-derived mesenchymal stem cells in a rabbit model. Stem Cells Transl Med 4: 1344-1351 (2015).

37. Ahmad Z, Wardale J, Brooks R, Henson E, Noorani A, Rushton $\mathrm{N}$. Exploring the application of stem cells in tendon repair and regeneration. Arthrosc 28: 1018-1029 (2012).

38. Gulotta LV, Kovacevic D, Ehteshami JR, Dagher E, Packer JD, Rodeo SA. Application of bone marrow-derived mesenchymal stem cells in a rotator cuff repair model. Am Sports Med 37: 2126-2133 (2009).

39. Lam E, Mok D. Open repair of massive rotator cuff tears in patients aged sixty-five years or over: is it worthwhile?. J Shoulder Elbow Surg 13: 517-521 (2004).

40. Uysal AC, Mizuno H. Differentiation of adipose-derived stem cells for tendon repair. Method Mol Biol 702: 443-451 (2011). 